of outpatient providers, and two established continuity of care measures (the usual provider of care index and the Bice-Boxerman continuity of care index) over 12 months. We used latent class analysis to categorize patients into classes based on overall, primary care (PCP)specific, gastroenterology (GI)-specific, and mental health specifichealthcare continuity patterns. RESULTS/ANTICIPATED RESULTS: The 26,751 Veterans in the analytic cohort had a mean of 13.3 (sd 8.63) office visits and 7.2 (sd 3.83) providers per patient. Patients were classified into five phenotypes: (1) moderate overall use and continuity; (2) low overall continuity of care; (3) high GI- and PCP-specific continuity of care; (4) low overall continuity of care with some mental health use; and (5) high utilization with substantial mental health use. In the subsequent year, 11,259 (42.1\%) patients had a hospitalization and 16,167 (60.4\%) had an emergency department visit. These groups varied in their sociodemographic and clinical characteristics, and in their risk for hospitalization and emergency department use. DISCUSSION/SIGNIFICANCE OF FINDINGS: Latent class analysis revealed five distinct primary and specialty care utilization patterns. Grouping patients with high intensity specialty care needs based on their propensity for healthcare continuity patterns can inform the development of personalized care navigation interventions.

66919

Third trimester electronic cigarette use and the risk of preterm birth, low birthweight, and small-forgestational age

Lin Ammar ${ }^{1}$ and Pingsheng Wu Ph.D. ${ }^{2}$

${ }^{1}$ Vanderbilt University Masters of Public Health Program;

${ }^{2}$ Vanderbilt University Medical Center

ABSTRACT IMPACT: Our study suggests that maternal e-cigarette use may reduce fetal growth and pose harm to newborns. OBJECTIVES/GOALS: Women are motivated to quit smoking during pregnancy. Many view electronic cigarettes (e-cigarettes) as a safer and healthier alternative to traditional tobacco smoke. We aim to determine the effect of third-trimester e-cigarette use on the risk of infant related outcomes. METHODS/STUDY POPULATION: We conducted a cross-sectional survey study using Pregnancy Risk Assessment Monitoring System (PRAMS). Women who gave live singleton births in 2016-2018 and at states that met response rate threshold criteria were included. Women were classified as never smokers, sole e-cigarette smokers, sole traditional cigarette smokers, and dual-users. Logistic regression was conducted to determine the association between third-trimester cigarette use and preterm birth ( $<37$ weeks), low birth weight ( $<2,500$ grams), and small for gestational age births (SGA, weight lower than the tenth percentile of the population). Analyses were weighted to account for the survey design and non-response. RESULTS/ ANTICIPATED RESULTS: 94,539 women (weighted population of $4,765,290$ ) were included. Compared with never smokers, thirdtrimester sole e-cigarette use increased the odds of preterm birth (Adjusted odds ratio [AOR]: 1.61, 95\% confidence interval [CI]: $1.05,2.48$ ), low birthweight (AOR: 1.49, 95\%CI: 1.06, 2.09), and SGA (AOR: 1.19, 95\%CI: 0.71, 2.00), sole traditional cigarette use increased the odds of preterm birth (AOR: 1.36, 95\%CI: 1.21, 1.52), low birthweight (AOR: 1.90, 95\%CI: 1.72, 2.10), and SGA (AOR: 2.28, 95\%CI: 2.05, 2.53); and dual use increased the odds of preterm birth (AOR: 1.17, 95\%CI: 0.82, 1.67), low birthweight (AOR: 2.16, 95\%CI: 1.58, 2.96), and SGA (AOR: 2.67, 95\%CI:
1.97, 3.64). DISCUSSION/SIGNIFICANCE OF FINDINGS: E-cigarette use, by itself or in combination with traditional cigarettes, increases the risk of preterm birth, low birthweight, and SGA. Our study suggests that maternal e-cigarette use may reduce fetal growth and pose harm to newborns.

69350

Using Control Charts to Inform Public Health and Community Engagement during the COVID-19 Pandemic Moira Inkelas ${ }^{1}$, Vladimir G. Manuel ${ }^{2}$, Iheanacho O. Emerewa ${ }^{1}$ and Tony $\mathrm{KuO}^{3}$

${ }^{1}$ Fielding School of Public Health, University of California Los Angeles; ${ }^{2}$ David Geffen School of Medicine, University of California Los Angeles; ${ }^{3}$ Los Angeles County Department of Public Health and Fielding School of Public Health and David Geffen School of Medicine, University of California Los Angeles

ABSTRACT IMPACT: Demonstrate applicability of an underutilized method for showing variation that enables public health agencies to respond to the COVID-19 pandemic OBJECTIVES/GOALS: Enacting sensible public policies in the coronavirus disease 2019 (COVID-19) pandemic requires real-time data that civic and public health leaders can easily interpret and act on. This collaboration between a CTSA and a local health department sought a novel use of control charts to provide timely and interpretable data. METHODS/STUDY POPULATION: Healthcare and other industries use control charts to understand the behavior of processes and systems so they can intervene on them. The CTSA science team developed statistical process control charts at the neighborhood level to help illustrate their value for decision-making as the pandemic progresses. This method included accounting for congregate populations (skilled nursing facilities, correctional facilities) to produce data for the general public. RESULTS/ANTICIPATED RESULTS: Patterns in COVID-19 vary over time by neighborhood. Juxtaposing control charts with social characteristics of local areas in a dashboard format provides granularity for decision-makers and data for engaging communities in changing behavior. Annotating time series charts in real time connects events and local knowledge with observed data, which can help authorities and people to learn and act based on variations displayed by the control charts about disease outbreaks and cases. School districts are among those that could benefit from control charts with information about the school community and how COVID-19 spread is occurring. DISCUSSION/SIGNIFICANCE OF FINDINGS: Control charts have rarely been used in public health despite their ease of use and interpretability. This study demonstrates a novel approach to providing timely, accurate data that can support real-time decision-making of government and public health as well as school districts, businesses, and others.

69567

Association between area deprivation index and longterm diabetic complications in a population of diabetic patients'

Riza C. Li, Kevin Ndura and Claudine T. Jurkovitz

Value Institute, ChristianaCare Health Systems

ABSTRACT IMPACT: To improve care and services for patients with chronic disease, health systems are focusing on evaluating social 
determinants of health of populations at risk; this information is currently not available in electronic health records (EHR) but we show that it could be accessed by linking area deprivation index to EHR. OBJECTIVES/GOALS: To inform care delivery and policy, health care systems are studying ways of improving social determinants of health $(\mathrm{SDoH})$ in patients with chronic disease such as diabetes (DM). Our goal was to better characterize the $\mathrm{SDoH}$ of a cohort of DM patients by using the area deprivation index (ADI). METHODS/STUDY POPULATION: Our study population included DM patients seen in primary care practices in 20132017. We integrated ADI levels to data extracted from electronic health records (EHR). ADI ranks neighborhoods by socioeconomic status calculated from income, education, employment and housing quality. ADI has 10 levels that we grouped into 5 categories of 2 levels. Addresses were geocoded using ArcMap to obtain census block groups information. We used multivariable logistic regression to calculate odds ratios (OR) and 95\% confidence intervals [], with diabetic complications as a binary dependent variable, ADI levels as the exposure, and demographics, smoking status and number of comorbidities as confounders. RESULTS/ANTICIPATED RESULTS: Our study population included 8,558 patients: $56 \%$ were female, $61 \%$ white, $31 \%$ black, $28 \%$ were on Medicare, $66 \%$ on commercial insurance, median age was 55 years, $57 \%$ never smoked, $10 \%$ had no comorbidities, $42 \%$ had 3 or more comorbidities, and $37 \%$ developed diabetic-related complications. After evaluating collinearity and adjusting for confounders, our multivariable analysis showed that worsening ADI was associated with higher likelihood of complications. Compared to ADI level 1\&2 (least disadvantaged), the ORs for patients residing in neighborhoods with ADI levels 3\&4, 5\&6, $7 \& 8,9 \& 10$ (most disadvantaged) were respectively $1.01[0.88$ 1.16), 1.20 [1.04-1.39], 1.15 [0.99-1.33], 1.30 [1.11-1.52]. DISCUSSION/SIGNIFICANCE OF FINDINGS: Neighborhood ADI could provide precious information to health care providers when associated to the EHR. We found that neighborhoods with ADI level 9\&10, which is not collected in the EHR, was significantly associated with a higher burden of disease. ADI could serve as a proxy for evaluating $\mathrm{SDoH}$.

78595

Assessing the influence of comorbidities in patients undergoing sternal reconstruction following cardiac surgery: a single institution's $\mathbf{1 5}$ year review

Edgar Soto, Pallavi A Kumbla, Ryan Restrepo, Thomas K Delay, Shadi K Awad, Sherry Collawn, Jorge de la Torre, Brad Denney, Jobe R Fix, John H Grant, Ali Kilic, Timothy W King, Prasanth Patcha, James Davies, Luis O Vasconez, Rene P. Myers

ABSTRACT IMPACT: Current practice guidelines offer a variety of treatment options for sternal reconstruction but complications and infections remain a serious surgical problem. This work seeks to provide a comprehensive picture of the com-morbidities and reconstructive methods that lead to success and improve patient outcomes. OBJECTIVES/GOALS: Patients that undergo cardiac surgery via the median sternotomy approach are at risk of wound complications that require repair. We seek to evaluate how outcomes of sternal reconstruction are influenced by patient comorbidities, flap usage and internal mammary artery grafts and methods of sternal closure. METHODS/STUDY POPULATION: We identified patients between 2005 and 2020 who underwent sternotomy followed by debridement and flap coverage at our institution. Comorbidities, method of reconstruction, demographic data, surgical history, and other factors pertaining to mortality and morbidity were collected. The data will then be analyzed to identify population characteristics using logistic regression variables to determine univariate and adjusted multivariable measures of association with mortality. We present the pre-liminary data analyzed using chi-square and oneway anova in R. RESULTS/ANTICIPATED RESULTS: In this study we present a preliminary characterization of one institution's sternal reconstruction patient outcomes with a variety of reconstruction methods including pectoralis advancement flaps, omental flaps and latissumus dorsi flaps. Notable preoperative comorbidities include $50 \%$ of patients $>$ age $60,18 \%$ with diabetes mellitus, 18 $\%$ with diagnosed hypertension, $18 \%$ with COPD, and $9 \%$ with a smoking history DISCUSSION/SIGNIFICANCE OF FINDINGS: In an evolving cardiothoracic landscape, clinical characteristics of patients being treated for sternal reconstructive surgery present a moving target. Understanding current risk factors, preoperative management and timing for aggressive surgical treatment offers an opportunity to update treatment protocol and maximize successful outcomes.

93137

\section{Interrogating cardio-protective MTSS1 variants in human} populations*

Megan F. Burke, Michael Morley, Yifan Yang, Theodore Drivas, Mingyao Li, Marilyn Ritchie, Thomas Cappola

Perelman School of Medicine, University of Pennsylvania

ABSTRACT IMPACT: It is our hope that a better understanding of the relationship between genetic variants that influence heart failure precursor traits will not only inform clinical care, but enable better assessment of inherited risk and will identify new biological targets for drug development. OBJECTIVES/GOALS: In this project, using a large-scale human genomic dataset with extensive phenotype data available, we intend to interrogate the known MTSS1 variants that have been associated with heart failure (HF) in previous GWAS studies in order to understand the directionality and mechanisms of their effects. METHODS/STUDY POPULATION: Data was obtained from the UK Biobank, a large prospective cohort of $\sim 500,000$ patients across the United Kingdom with extensive phenotype data, including $\sim 50,000$ patients with cardiac MRI and 200,000 with whole exome sequencing. We test for associations between genetic variants at the MTSS1 locus and HF precursor traits using logistic regression or linear regression, adjusting for age, gender, and principal components (PCs) of ancestry. For rare variant analyses we 'bin' rare variants $(\mathrm{MAF}<0.01)$ using the software tool BioBin to aggregate low frequency genetic variants into single genetic units. RESULTS/ANTICIPATED RESULTS: Preliminary data have shown that variants in the known MTSS1 enhancer region which reduce MTSS1 expression are associated with smaller, more contractile hearts. We anticipate that common variants known to reduce enhancer activity will attenuate heart failure precursor traits, will be associated with a reduced risk clinical heart failure, and will favorably impact clinical outcomes once HF is established. We also anticipate that rare exonic variants predicted to impair MTSS1 function 